\title{
Analysis on the Influencing Factors of Population Agglomeration under the Background of Urbanization: A Case Study of Xinjiang Production and Construction Corps (XPCC)
}

\author{
Li Sheng, Zhu Xiaoling \\ College of Economic and Management, Tarim University, lear Xinjiang,843300, China
}

\begin{abstract}
Keywords: Xinjiang Production and Construction Corps (XPCC); Town population; Agglomeration
\end{abstract}

\begin{abstract}
Population agglomeration is the core task of urbanization promotion. XPCC's population is small with low concentration, which is not conducive to the realization of the goal of leapfrogging development and the historical mission of reclaiming and garrisoning the frontier. Accelerating population agglomeration is the first and key step of urbanization promotion. Based on the analysis of the characteristics and influencing factors of urban population concentration in XPCC, the author puts forward corresponding countermeasures and suggestions.
\end{abstract}

\section{Introduction}

Speeding up the process of urbanization is a major strategic arrangement made by the central government to better develop and strengthen the Corps under the new historical conditions and to play a better role in the Corps. The main body of urbanization is human, which is the agglomeration of human production and life activities in the regional space. In the process of realizing the industrial and spatial transfer of human population, the modernization of population development is realized through optimizing people's production and living environment. The urbanization of military regiment is a process of realization, which is characterized by gathering population, focusing on industrial transformation, taking social transformation as goal and taking reclamation and frontier defense as its duty.

\section{The significance of population agglomeration to the development of urbanization in XPCC}

2.1 Population agglomeration is an inevitable trend of XPCC following the development of cities and towns

Population agglomeration refers to the phenomenon and process in which people of different ages, sexes and occupations converge in a particular geographical area from the desired economic, environmental, social and psychological effects, which is a socio-economic phenomenon from sparse to dense. The second and third industries generally have higher factor productivity than the previous one, and the large cities generally have higher factor productivity than the small and medium-sized cities, which thus makes the rural areas more urbanized. The evolution of cities is from small and medium-sized cities to large cities, a single city to urban agglomeration and continuous urban continuous development. The primary function of urbanization is agglomeration, which realizes the optimal allocation of social resources while realizing the agglomeration of population to towns. Urbanization is the inevitable trend of economic and social development and the progress of human civilization, where people are engaged in economic, political, cultural and other activities in the pursuit of high efficiency and good benefits, while cities and towns makes it possible for people to do these activities in cities and towns more efficiently and more effectively than in the countryside because of their inherent clustering effect and the resulting unparalleled advantages of the countryside. All kinds of resources, factors and activities of industry, population and economic, political and cultural activities are always concentrated in cities and towns, which will inevitably promote the process of urbanization. 


\subsection{Population agglomeration is the objective requirement of population security in XPCC}

At the beginning of the urbanization Development Office, the total population of the Corps was about 170,000. In 1964, the population of the Corps was pictured at 1 million and it broke through the threshold of 2 million in 1972. In the nearly 40 years since 2010, the population of the Corps has been hovering around 2.5 million people while the growth rate has been slow. During the seven-year period from 2010 to 2017, the population growth rate of the Corps fluctuated. On the one hand, the reason is that the population growth of Bingtuan is stable in the low fertility level for a long time, whose total fertility rate of women is about 1.2, which is lower than the fertility replacement level. On the other hand, the population of Bingtuan mainly depends on mechanical growth, but due to the income level, living environment, land quantity and polices and so on, the number of immigrants decreases while the number of emigrants increases. In addition, many local residents go out for development and move out more than they move in, resulting in a massive loss of population. The above three reasons lead the population growth rate to slow down, reflecting the pattern of population growth from mechanical growth to slow natural growth. The "short board" of urbanization is an important factor leading to the slow growth of population and the prominent problem of population security., which is unable to provide workers with a good environment, higher income and more job, which make the workers and staff "guest mentality" serious, lack of a sense of belonging at home, resulting in a large number of population loss, staff and workers unstable and quality decline.

\section{The present situation of population agglomeration in the towns of XPCC}

From the view of urban population scale, Shihezi City is the first city of Bingtuan. After decades of development, Shihezi City has become a medium-sized city, whose total population of Alar, Tumuke and Wujiaqu are more than or close to 100,000 people. However, its urban population concentration is not high because most of the population is scattered in the surrounding farms. In addition to the high population density of Shihezi, other cities have a small population density and sparse population with less than 200 people each square kilometer. From 2000 to 2016, the average annual population growth rate of cities is only 0.01-0.02 which means that the population growth rate is slow. From the view of the population scale of small towns in Tuanchang and the overall distribution is scattered with poor agglomeration.

\section{Characteristics of urban population concentration in XPCC}

\subsection{Patterns of coexistence and agglomeration of local and foreign residents}

Bingtuan town population agglomeration has the characteristics of the combination of nearby urbanization and urbanization in different places. Local urbanization is to change the former farm management mode into the urban community management mode through the town to provide good infrastructure, beautiful living environment and public service level, letting the workers enjoy the civilized way of life of the town. Urbanization refers to the transfer of company workers into the town and live in concentrated, while attracting mainland and Xinjiang college graduates, demobilized soldiers, migrant workers and the surrounding rural residents to live in the town of Bingtuan. For the small towns of the Bingtuan farm, the emphasis is to realize the concentrated residence of the company staff and workers to the towns and mainly solve the problem of population agglomeration into the towns within the Bingtuan; For the Bingtuan cities, the emphasis is on absorbing the highly qualified population outside the Bingtuan, including the hinterland and Xinjiang (College graduates, re-servicemen, etc.) and the goal is to improve the mechanical growth rate of population, to undertake the main task of gathering foreign population and thus to ensure that the proportion of population in the autonomous region not reduced, ato achieve the goal of 2.8 million population scale proposed by the 12th Five-Year Plan of the Corps. 


\subsection{Agglomeration patterns of residential areas and working areas}

Agriculture has regional characteristics, and the scattered residence of labor force is the objective requirement of agricultural farming mode. Agriculture is the pillar industry of the Corps. The Bingtuan has 1 million hectares of arable land and 400,000 agricultural front-line labors, which are scattered among the 13 agricultural divisions and 1998 companies of the corps. The urbanization strategy of the Corps proposes to concentrate the company population within 10 kilometers from the regiment to the regiment, which shows that the majority of the sub-companies will change from the dual function of undertaking production and living bases to the functions of only assuming short-term residence and temporary operating points. Formed "production in rural areas, living in towns", residential areas and work apart from the "amphibious" model. At present, over $80 \%$ of the company staff and workers in the reclamation area of the eighth Agricultural Division have entered the town of Tuanchang to live, and other divisions are active in its promotion. This model is only the transition model of the initial stage of Bingtuan town. With the rapid development of agricultural modernization, industrialization and service industry, the "amphibious" population will be completely liberated from agriculture and engaged in. The third industry produces finally realizes the residence and the employment space integration.

\section{An Analysis of the factors affecting the Urban population concentration in XPCC}

\subsection{Impact of population agglomeration platforms}

Bingtuan is a special organization of the integration of party, government and military, but the function of "government" cannot be fully reflected. The main body of the management of urban construction is the government. The government meets the needs of urban construction and people's life through town finance. In addition to the four cities, the division and regiment is not a first-level government, nor is a first-level government authorized organization, and there is no financial administration, industry and commerce, taxation and other government management authority, which means Tuanchang town is only a natural town (except Beiquan town). It has distinct characteristics of "running towns by enterprises", whose main body of investment and management of urban construction are absent while the infrastructure and towns are weak, the environment cannot be improved while the burden of workers and staff is heavy, which results in the staff and workers unwilling to settle down for a long time and the difficulty of population agglomeration.

\subsection{Impact of production methods}

The effect of agri-oriented farming on urban population agglomeration is completely different from that of industrial and commercial production. The scattered population is adapted to the mode of production in which farming is the main form of production. The development of the three industries requires a relatively concentrated population. The third industry is the power source of urban population agglomeration. With the rapid development of state-level economic and technological development zones, autonomous regions, Bingtuan economic development zones, the presence of large enterprises and large groups, it will inevitably bring huge flows of people, logistics and information, which can accelerate the population agglomeration from the interior and the surrounding areas of Xinjiang to the city of Xingtuan. With the acceleration of the process of agricultural industrialization and the development of tourism, service industry, real estate and other tertiary industries, the agglomeration of population to small towns will be accelerated.

\subsection{Impact of the level of agricultural modernization}

The level of agricultural modernization is the motive factor for the transfer of agricultural surplus labor force to cities and towns. Bingtuan agricultural mechanization is in the leading level of the whole country, while the machine tillage, the machine seeding and the machine harvest level have reached 92th. Except the cotton flower harvest that has not fully implemented the mechanization, the others have realized the mechanization. The party committee of the Corps has proposed that at present and for a period to come and the Corps will give full play to its advantages 
in agriculture, vigorously raising the level of agricultural modernization and making it a vanguard in leading the development of modern agriculture in Xinjiang and throughout the country. With the rapid progress of agricultural modernization, the popularization of modern farming methods and management model, a large number of surplus labor force is liberated from the field agriculture, and it is possible to enter the town from the secondary and tertiary industries.

\subsection{Effects of planting structure}

Planting structure is an important factor that affects workers' agglomeration into cities and towns. Under the condition that the staff and workers of Bingtuan have not got rid of the condition of dependence on agriculture, after living in concentrated cities and towns of the team workers, they are far away from the land contracted by their families, and different planting structures have different effects on the population to the towns. The grain-based planting structure has a higher degree of mechanization with less labor consumption and a strong willingness to gather people into cities and towns. Cotton planting structure is the main business with other crops in cotton field and more labor costs, while there are a lot of regular farm work in the year and most of the time will be spent in cotton planting; Garden forestry also needs a lot of labor input; Facility agriculture is less affected by seasonality, working in the fields for many years, these planting structures lead to the labor population agglomeration to the town is not a very strong will.

\subsection{Effects of spatial distances from towns}

Distance is a significant factor affecting the flow. The long and short flow distance not only reflects the size of transportation cost, but also affects the transmission of information in the labor market. The farther the distance is, the greater the uncertainty of information acquisition is, and the higher the risk of mobility is. The pressure of psychological pressure on the flow decision-making resistance will also thus increase while the flow of expected gains will be reduced. Because the population agglomeration to the town is mainly driven by the administrative power of the regiment, the distance can reduce the psychological expected impact of the population agglomeration to a certain extent, but the impact of distance is still there. General distance town(City)from relatively far away from the company, workers to the town gathering costs are relatively high, which is a mainly higher transportation costs. The pattern of "living idle in cities and towns and living in busy working places" will bring inconvenience to the production of workers and staff.

\subsection{Impact of migration costs}

Migration costs are mainly twofold: On the one hand, the population agglomeration of the Corps is not restricted by household registration and social security. General rural population to urban migration is from the low level of rural welfare to the high level of urban migration, where there is a problem of urban social security sharing, which is also the root of the existence of migrant workers. And Bingtuan workers with their families and children are urban census register and the migration to the regiment is not subject to household registration restrictions, where workers and staff enjoy basic social security and floating workers can enjoy social security problems. On the other hand, the population agglomeration must involve land demolition and other issues. Compared with the local countryside, the land of the Bingtuan farm is state-owned land while the local countryside is the land under collective ownership. In the process of population concentration and land resources integration, the cost borne by the Corps is obviously lower than that of the local rural areas.

\section{Conclusions}

It can be seen that the current Xinjiang Bingtuan is facing the economic and social urbanization problems that are more complex. The development mode of urbanization of Bingtuan is not only different from the development mode of foreign countries, but also different from the urbanization road of other areas in China, even when compared with the urbanization mode of Xinjiang Uygur Autonomous region. In the construction of urbanization in the future, the Corps should pay attention to the combination of seeking truth from facts and the courage to innovate, and constantly 
explore the road of construction suitable for its own development in practice.

\section{Acknowledgements}

Fund Project: National Philosophy and Social Science Fund Project (Project Number: 12XJY004)

\section{References}

[1] Chu Hongxia. Analysis on the Construction and Development Characteristics of Xinjiang Production and Construction Corps and Heilongjiang Reclamation Cities and Towns[J]Agricultural Archaeology 2016(06)88-95.

[2] Zuo Haixia, Li Wan-ming. Evaluation and Development Strategy of Urbanization Construction in Xinjiang Bingtuan[J]Journal of Shanxi Agricultural University.

[3] Bai Yan, Yin Xiao-.A study on Urban Population Agglomeration in Xinjiang Bingtuan[J]Xinjiang Farming and Reclamation Economy(02)66-72.

[4] Gao Gang Cang, Jia Ningning. Strengthening the Management of the Bingtuan with the Urbanization of Xinjiang[J]People's Forum 2016(02)224-225.

[5] Tang Chao, Hu Yi-ting. Study on the Effect of Modern Agriculture on Promoting New Urbanization: a case study of Xinjiang Bingtuan[J]Xinjiang farming economy (09)25-32. 